\title{
Surgery for atrial fibrillation in patients with mitral valve disease: Results at five years from the International Registry of Atrial Fibrillation Surgery
}

Joao Melo, MD, PhD, ${ }^{a}$ Teresa Santiago, MSc, ${ }^{a}$ Carlos Aguiar, MD, ${ }^{a}$ Eva Berglin, MD, PhD, ${ }^{b}$ Michael Knaut, MD, ${ }^{c}$ Ottavio Alfieri, MD, PhD, ${ }^{d}$ Stefano Benussi, MD, PhD, ${ }^{d}$ Haw Sie, MD, ${ }^{e}$ Mathew Williams, MD, ${ }^{f}$ Fernando Hornero, MD, PhD, ${ }^{g}$ Giuseppi Marinelli, MD, ${ }^{\mathrm{h}}$ Paul Ridley, MD, Enrique Fulquet-Carreras, MD, and António Ferreira, MD ${ }^{\mathrm{a}}$

See related editorial on page 727.
From the Hospital Sta Cruz, ${ }^{a}$ Carnaxide, Portugal; Sahlgrenska University Hospital, ${ }^{\mathrm{b}}$ Goteborg, Sweden; Dresden University Hospital, ${ }^{\mathrm{c}}$ Dresden, Germany; San Raffaele Hospital, ${ }^{\mathrm{d}}$ Milano, Italy; Isala Klinieken, ${ }^{\mathrm{e}}$ Zwolle, The Netherlands; Columbia University Medical Center, ${ }^{\mathrm{f}}$ New York, NY; Hospital General Universitario de Valencia, ${ }^{\text {g }}$ Valencia, Spain; Policlinico S. Orsola, ${ }^{\text {h }}$ Bologna, Italy; North Staffordshire Royal Infirmary, ${ }^{\mathrm{i}}$ Staffordshire, United Kingdom; and Hospital Universitario de Valladolid, ${ }^{\mathrm{j}}$ Vallodolid, Spain.

Read at the Eighty-sixth Annual Meeting of The American Association for Thoracic Surgery, Philadelphia, Pa, April 29-May 3, 2006.

Stefano Benussi reports consulting fees from Estech; lecture fees from St Jude, Medtronic, and Cryocath; and an educational grant from Atricure.

Received for publication April 28, 2006; revisions received June 25, 2007; accepted for publication Aug 30, 2007.

Address for reprints: Joao Melo, MD, PhD, Hospital de Santa Cruz, Av. Prf. Reynaldo dos Santos, 2799-523 Carnaxide, Portugal (E-mail: jmelo@hsc.min-saude.pt).

J Thorac Cardiovasc Surg 2008;135:863-9 $0022-5223 / \$ 34.00$

Copyright (C) 2008 by The American Association for Thoracic Surgery

doi:10.1016/j.jtcvs.2007.08.069
Objectives: We sought to assess the clinical and survival benefit of atrial fibrillation surgery in patients submitted to mitral valve surgery after stabilization of postoperative rhythm at 1 year.

Methods: One thousand seven hundred twenty-three patients were enrolled. Patients with follow-up of longer than 1 year $(n=972)$ were divided into 3 groups according to surface electrocardiographic rhythm during follow-up visits: stable sinus rhythm, stable atrial fibrillation, and intermittent rhythms. Adverse cardiac event incidence and predictors of long-term outcome were compared among the 3 groups.

Results: In-hospital mortality was $2.6 \%$. Risk factors for mortality were the cut-andsew technique (odds ratio, 8.92; 95\% confidence interval, 1.71-46.50; $P=.009$ ) and isolated left atrial procedure (odds ratio, 0.16 ; $95 \%$ confidence interval, 0.04-0.56; $P=.004)$. At 1 year, $63.4 \%$ patients were in stable sinus rhythm. Stable sinus rhythm was found to be associated with early and late survival $(P=.01$, log-rank analysis). Multivariate binary logistic regression analysis found that left atrial dimension (odds ratio, $0.97 ; 95 \%$ confidence interval, $0.96-0.99 ; P=.005$ ) and concomitant coronary revascularization (odds ratio, $0.48 ; 95 \%$ confidence interval, $0.25-0.92 ; P=.027$ ) were independent predictors of stable sinus rhythm at 1 year after surgical intervention. At 48 months' follow-up, predictors for stable sinus rhythm were biatrial surgical approach and absence of preoperative permanent atrial fibrillation (odds ratio, 3.56; 95\% confidence interval, 1.62-7.83; $P<.002$ ). Left atrial size (each millimeter) has a borderline statistical significance (odds ratio, 0.97 ; $95 \%$ confidence interval, $0.93-1.00 ; P=.065)$. Thromboembolic events were found to be associated with absence of stable sinus rhythm $(P=.010, \log$-rank analysis $)$.

Conclusions: The achievement of stable sinus rhythm is a predictor of better survival and lower incidence of thromboembolic events. Predictors of stable sinus rhythm were smaller dimensions of the left atrium, biatrial approach, absence of preoperative permanent atrial fibrillation, and absence of concomitant coronary artery bypass grafting.

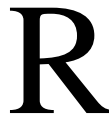
ecent reports of outcomes after atrial fibrillation treatment with mitral valve surgery have been limited by the lack of a clear definition of patient selection criteria and of consistent data on long-term results. ${ }^{1}$

Heart rhythm instability after these procedures is frequent. Nomenclature for these different rhythms is not uniform, and results are often described as "out of atrial fibrillation" or "regular rhythms," which have led to some misleading conclusions. ${ }^{1,2}$ Long-term use of concomitant antiarrhythmic medication after surgical intervention is seldom reported.

The International Registry for Atrial Fibrillation Surgery (RAFS) covers a large cohort of patients submitted to atrial fibrillation surgery in different clinical contexts. 


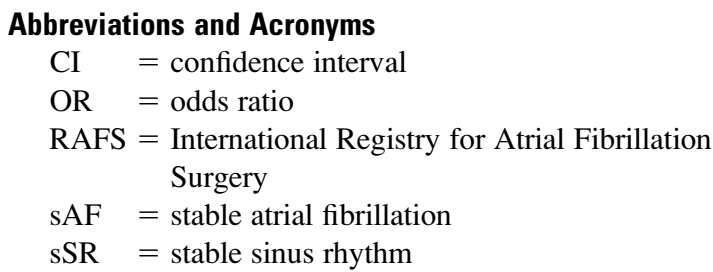

RAFS uses a common nomenclature for clinical variables and a standardized reporting method for the surgical procedure and clinical outcomes.

The aim of this study was to assess the clinical and survival benefit, if any, of sinus rhythm recovery in the subset of patients submitted to mitral valve surgery included in the RAFS. Safety, efficacy, and benefits of atrial fibrillation surgery were evaluated on short- and long-term data. Late use of antiarrhythmic therapy was also assessed.

A classification based on heart rhythm stability after mitral valve surgery was used. The effect of recovery of stable sinus rhythm (SSR) on patient survival and on major adverse cardiac events was studied.

\section{Materials and Methods}

Of 2140 patients enrolled in the RAFS, 1723 were submitted to concomitant mitral surgery. Data from these patients were collected retrospectively from clinical reports between December 2003 and March 2006. Patients were submitted to surgical intervention in 10 hospitals located in Europe and America enrolled in the RAFS. Criteria used for the definition and classification of atrial fibrillation were those recommended by the European Society of Cardiology, American Heart Association, and American College of Cardiology. ${ }^{3}$

A total of 56 variables were included concerning demographic and clinical data at discharge and during follow-up. Common accepted nomenclature for the reported variables can be viewed at www.registryafsurgery.com, with the most significant ones being described in Table 1.

A standard protocol for valve or coronary surgery was used. All patients were operated on during cardiopulmonary bypass, according to local recommendations. Surgical techniques used to treat atrial fibrillation and left atrial appendage closure were at the physicians' discretion (Table 2). In 283 (16.4\%) patients the left atrial appendage was left open.

Independent prognostic factors for in-hospital mortality were determined for the total group of patients. Follow-up outcomes are based on data from patients who have a minimum of 1 year's follow-up ( $\mathrm{n}=972$ ). The average follow-up for these patients was $29.0 \pm 21.4$ months (median, 23 months; interquartile range 26.4 months).

In each of the 972 patients, rhythm was determined at every follow-up visit according to the institutional protocol. Three institutions used 24-hour Holter monitoring in the first 6 months and electrocardiography at each follow-up visit thereafter, 1 institution used either method throughout the follow-up period according to

TABLE 1. Data from all patients according to concomitant cardiac procedures

\begin{tabular}{|c|c|c|c|c|c|c|c|}
\hline Groups & All $(n=1723)$ & $M(n=925)$ & $M, A(n=205)$ & $M, T(n=259)$ & $M, C(n=86)$ & $M, A, T(n=71)$ & $M, 0(n=179)$ \\
\hline \multicolumn{8}{|l|}{ Age (y) } \\
\hline Mean & 63 & 62 & 66 & 64 & 69 & 63 & 66 \\
\hline SD & 11 & 11 & 10 & 11 & 7 & 13 & 10 \\
\hline \multicolumn{8}{|l|}{ Sex } \\
\hline$\%$ Male & 39.5 & 38.5 & 38.5 & 28.6 & 73.3 & 25.4 & 51.0 \\
\hline \multicolumn{8}{|l|}{ Type of AF (\%) } \\
\hline Paroxysmal & 11.8 & 12.5 & 15.9 & 7.4 & 14.5 & 12.9 & 11.7 \\
\hline Persistent & 11.6 & 12.4 & 18.3 & 8.1 & 24.1 & 2.9 & 7.3 \\
\hline $\begin{array}{c}\text { Permanent } \\
\text { AF duration (v) }\end{array}$ & 76.6 & 75.1 & 65.9 & 84.5 & 61.4 & 84.3 & 81.0 \\
\hline Mean & 6.6 & 6.7 & 7.6 & 7.1 & 4.6 & 5.3 & 6.1 \\
\hline SD & 6 & 6 & 7 & 7 & 5 & 5 & 6 \\
\hline \multicolumn{8}{|l|}{ Cause (\%) } \\
\hline Rheumatic & 50 & & & & & & \\
\hline Degenerative & 19 & & & & & & \\
\hline Ischemic & 3 & & & & & & \\
\hline Other & 28 & & & & & & \\
\hline Previous TE & 13 & 13 & 13 & 9 & 6 & 10 & 11 \\
\hline \multicolumn{8}{|l|}{ Size of LA $(\mathrm{mm})^{*}$} \\
\hline Mean & 57 & 58 & 56 & 57 & 52 & 55 & 54 \\
\hline SD & 22 & 28 & 8 & 11 & 8 & 9 & 9 \\
\hline Mode & 50 & 50 & 60 & 50 & 50 & 53 & 50 \\
\hline
\end{tabular}

$M$, Mitral; $A$, aortic; $T$, tricuspid; $C$, coronary; 0 , other; $S D$, standard deviation; $A F$, atrial fibrillation; $T E$, thromboembolic events. ${ }^{*}$ Echocardiographic measurements were made according to each institution's protocol. 
TABLE 2. AF procedures

\begin{tabular}{lrr}
\hline & N & $\%$ \\
\hline Instrument type & & \\
Ablation & 1679 & 97 \\
Knife & 44 & 3 \\
Type of ablation & & \\
Dry unipolar RF & 655 & 38 \\
Irrigated unipolar RF & 365 & 21 \\
Dry bipolar RF & 108 & 6 \\
Microwave & 462 & 27 \\
Argon cryoablation & 65 & 4 \\
Other & 68 & 4 \\
Atrial approaches & & 16 \\
Maze III & 269 & 19 \\
Non-maze Biatrial & 335 & 65 \\
Left side only & 1117 & 0. \\
Right side only & 2 & \\
Left-sided techniques & & \\
$\quad$ (excluding maze) & & 2 \\
BIPV & 1076 & 15 \\
Linear connection PV & 127 & \\
Division of LA & 219 & \\
Others & 30 &
\end{tabular}

$R F$, Radiofrequency; $B I P V$, bilateral isolation of pulmonary veins; $P V$, pulmonary veins; $L A$, left atrium.

the case, and the remaining used 12-lead electrocardiographic recordings.

Patients were divided into 3 groups according to the rhythm documented during the first year: sSR, stable atrial fibrillation (sAF), and intermittent rhythms. sSR was defined as sinus rhythm at all follow-up visits during the first year.

A similar criterion was adopted for sAF. All other rhythms were described as intermittent rhythms. Atrial flutter rhythm was considered as SAF for analysis purposes.

Adverse cardiac event incidence and predictors of long-term outcome were compared in the 3 groups.

Summary statistics were presented as frequencies and percentages, means \pm standard deviation, medians, and interquartile ranges. Missing data were not defaulted to negative, and denominators reflect only actual reported cases.

Associations of outcomes among nominal variables were compared by using the $\chi^{2}$ test with the Yates correction or the 2-sided Fisher exact test, when appropriate. Bivariate comparisons of continuous variables were investigated with the Student $t$ test.

Survival analyses with Kaplan-Meier methods were performed to estimate survival and freedom from thromboembolic events. Cox proportional hazards models were used to identify the predictors of early and late mortality and the predictors of maintenance in $\mathrm{SSR}$.

Iterative logistic modeling was performed for sSR by using the likelihood ratio test for model selection.

\section{Results}

In-hospital mortality for the 1723 patients was $2.6 \%(n=45$; $99 \%$ confidence interval $[\mathrm{CI}], 1.7 \%-3.9 \%)$ Independent
TABLE 3. Hospital morbidity

\begin{tabular}{lr}
\hline \multicolumn{1}{c}{ Type } & $\mathbf{N}$ \\
\hline Cardiac & 139 \\
Lung & 1 \\
GI & 23 \\
CVA/TIA & 4 \\
Renal & 16 \\
Infectious & 8 \\
Miscellaneous & 9
\end{tabular}

GI, Gastrointestinal; CVA/TIA, cerebral vascular accident/transient ischemic attack.

predictors for in-hospital mortality were the use of the cutand-sew technique (odds ratio [OR], 8.92; 95\% CI, 1.7146.50; $P<.009)$ and an isolated left atrial procedure $(\mathrm{OR}$, 0.16; 95\% CI, 0.04-0.56; $P=.004$ ).

In-hospital morbidity occurred in $153(9 \%)$ patients (99 $\%$ CI, 7\%-11\%). These patients experienced 217 complications (Table 3), an average of 1.4 complications per patient.

The mean hospital stay was $12.4 \pm 10$ days. At discharge, $59 \%$ of patients $(\mathrm{n}=971)$ were in sinus rhythm, $34 \%(\mathrm{n}=$ 554) were in atrial fibrillation, $2.6 \%(\mathrm{n}=42)$ were in atrial flutter, and the remaining $4.4 \%$ had other rhythms $(n=99$; ie, nodal, sick sinus, and atrial heart rhythms). Of the 52 patients who required pacemaker implantation before discharge, 20 (1.4\%; 95\% CI, 0.9\%-2.2\%) were submitted to left-sided approaches, and $32(6 \%$; 95\% CI, 4.0\%-8.0\%) were submitted to biatrial approaches $(P<.001)$.

Patients with follow-up of less than 1 year were excluded from midterm analysis. Survival and embolic event analysis were performed in the remaining 972 patients.

Rhythm at 1 year was documented by means of electrocardiography in 869 patients by using Holter monitoring in 101 patients and by means of intracavitary recordings in 2 patients.

Biatrial approaches were used in 307 of 972 patients, and follow-up data at 1, 2, 3, and 4 years were available in 244 , 104, 94, and 59 subjects, respectively.

At 1 year, $66 \%$ of patients were in sinus rhythm, $2.72 \%$ were in atrial flutter, $25 \%$ were in atrial fibrillation, and $6.3 \%$ had other rhythms (atrial, nodal, and pacing).

One hundred fourteen (6.6\%) patients died during followup, and 144 adverse cardiac events were found in 120 (14.5\%) patients. Considering the patient subset with longterm follow-up ( $>48$ months, $\mathrm{n}=181)$, the attrition rate for losing SSR was $3 \%$ per year.

sSR was found to be associated with higher early and late survival $(P=.01, \log$-rank analysis) when compared with all other heart rhythms (Figures 1 and 2).

The use of antiarrhythmic therapy was similar in both groups in early and long-term follow-up (Table 4). At 4 years, $34 \%$ of patients with sSR versus $21 \%$ of patients with $\mathrm{sAF}$ were taking antiarrhythmic medication. 


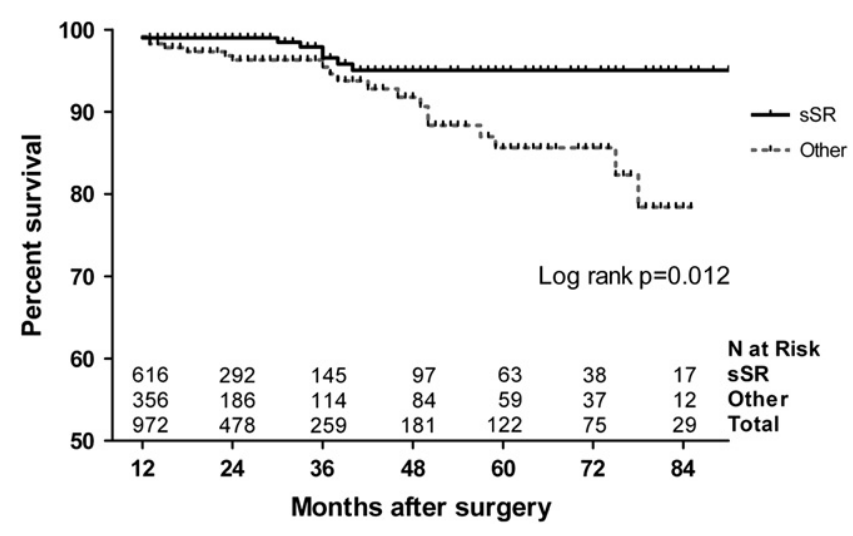

Figure 1. Actuarial survival after the first year of follow-up for stable sinus rhythm (sSR) versus other rhythms.

Multivariate binary logistic regression analysis (see Table 5) found that at 1 year after surgical intervention, left atrial dimension was an independent predictor for SSR at 1 year after surgical intervention (OR, 0.97 for each millimeter; $95 \%$ CI, 0.96-0.99; $P=.005$ ). Concomitant coronary revascularization was found to be an independent negative predictor of sSR (OR, 0.48; 95\% CI, 0.25-0.92; $P=.027$ ).

In the subset of patients with more than 48 months' follow-up, multivariate analysis (see Table 5) identified the biatrial surgical approach (OR, 5.87; 95\% CI, 1.94-17.74; $P=.002)$ and the absence of permanent atrial fibrillation (OR, 4.00; 95\% CI, 1.06-14.29; $P=.041$ ) as significant predictors for sSR at 4 years. Left atrial size had a borderline statistical significance when considered as a continuous variable (OR, 0.97 for each millimeter; 95\% CI, 0.93-1.00; $P=.065$ ) but reached statistical significance when dichotomized (OR, 0.39 for left atrium $>55 \mathrm{~mm} ; 95 \%$ CI, $0.19-0.84 ; P=.015$; the best cutoff value was determined by means of receiver operating curve analysis).

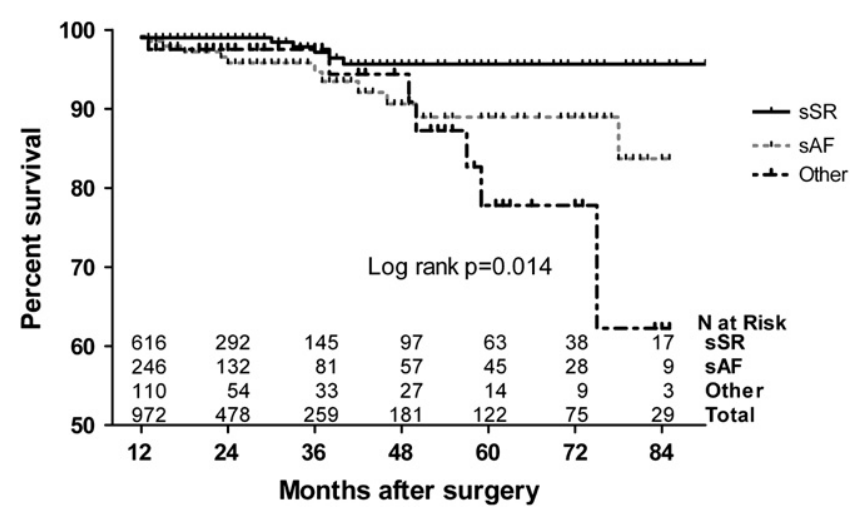

Figure 2. Actuarial survival after the first year of follow-up according to stable sinus rhythm (sSR) versus stable atrial fibrillation (sAF) versus "other."
TABLE 4. Antiarrhythmic therapy

\begin{tabular}{llllll}
\hline & sSR & & & \multicolumn{2}{c}{ sAF } \\
\cline { 6 - 6 } Year & $\%$ & $\mathbf{9 5 \%} \mathbf{~ C l}$ & & $\%$ & $\mathbf{9 5 \%} \mathbf{~ C l}$ \\
\hline 1 & 34 & $30-38$ & 30 & $26-34$ \\
2 & 30 & $25-37$ & 24 & $17-32$ \\
3 & 41 & $31-49$ & 31 & $21-43$ \\
4 & 34 & $24-47$ & 21 & $11-35$ \\
5 & 54 & $38-69$ & 20 & $10-36$ \\
\hline
\end{tabular}

$s S R$, Stable sinus rhythm; $s A F$, stable atrial fibrillation.

Twenty-five (3\%) patients experienced thromboembolic events. Thromboembolic events were found to be associated with absence of sSR $(P=.010, \log -$ rank analysis; Figure 3$)$.

\section{Discussion}

Atrial fibrillation surgery in patients undergoing mitral valve operations has become very common in the last decade. Alternative techniques to the maze procedure were developed over the years in an attempt to avoid its complexity while retaining effectiveness. ${ }^{4}$ Concomitantly, ablation techniques to create atrial scars were used as an alternative to the classic cut-and-sew technique. ${ }^{5-8}$ Despite these advances, the clinical benefit of atrial fibrillation surgery and the best technique to perform it remain unclear.

It is well known that sinus rhythm recovery is not always achieved. Moreover, a significant number of patients can

TABLE 5. Predictors for stable sinus rhythm at 1 and 4 years

\begin{tabular}{|c|c|c|c|}
\hline Variable & OR & $P$ value & $95 \% \mathrm{CI}$ \\
\hline \multicolumn{4}{|l|}{$1 \mathrm{y}$} \\
\hline Age (each year) & 0.98 & .008 & $0.96-0.99$ \\
\hline Female sex & 1.05 & .806 & $0.71-1.55$ \\
\hline Permanent AF & 0.68 & .139 & $0.40-1.14$ \\
\hline Knife vs ablation & 1.10 & .829 & $0.48-2.53$ \\
\hline Biatrial approach & 0.93 & .722 & $0.64-1.37$ \\
\hline Left atrial size (each mm) & 0.97 & .005 & $0.96-0.99$ \\
\hline \multicolumn{4}{|l|}{ Concomitant surgery } \\
\hline Aortic & 0.87 & .646 & $0.48-1.57$ \\
\hline Tricuspid & 0.69 & .083 & $0.45-1.05$ \\
\hline Coronary & 0.48 & .027 & $0.25-0.92$ \\
\hline \multicolumn{4}{|l|}{4 y } \\
\hline Age (each year) & 0.99 & .541 & $0.95-1.03$ \\
\hline Female sex & 1.29 & .578 & $0.52-3.20$ \\
\hline Permanent AF & 0.25 & .041 & $0.07-0.94$ \\
\hline Knife vs ablation & 0.38 & .059 & $0.14-1.04$ \\
\hline Biatrial approach & 5.87 & .002 & $1.94-17.74$ \\
\hline Left atrial size (each mm) & 0.97 & .065 & $0.93-1.00$ \\
\hline \multicolumn{4}{|l|}{ Concomitant surgery } \\
\hline Aortic & 0.52 & .470 & $0.09-3.10$ \\
\hline Tricuspid & 0.64 & .490 & $0.18-2.28$ \\
\hline Coronary & 0.36 & .170 & $0.09-1.54$ \\
\hline
\end{tabular}

Results are from binary logistic regression analysis. $O R$, Odds ratio; $\mathrm{Cl}$, confidence interval; $A F$, atrial fibrillation. 


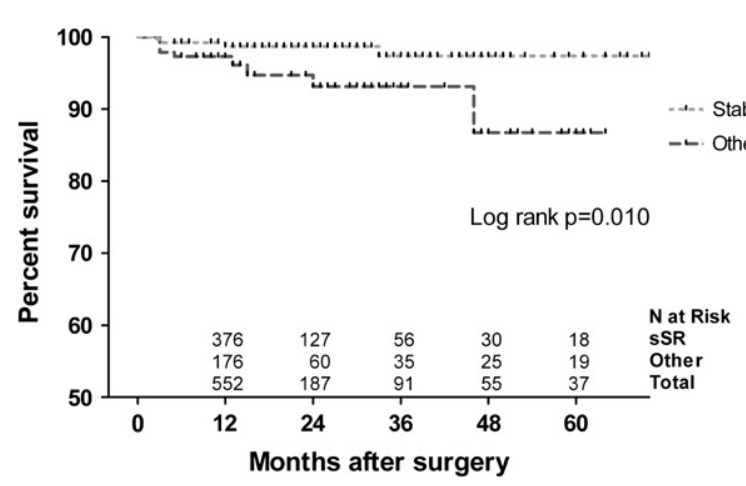

Figure 3. Survival free of thromboembolic events according to rhythm. sSR, Stable sinus rhythm.

change rhythm over time, mainly in the first 6 months after surgical intervention, probably as a result of the healing process, neurohumoral modulation, or both. ${ }^{9-12}$ Classifying these patients based on their discharge rhythms makes them behave as crossovers and creates confusion in outcome assessment.

The use of a clear definition of sSR in the first year after mitral surgery enabled us to assess the clinical outcome of atrial fibrillation surgery in those patients in whom it is truly effective (nearly two thirds in this registry) and identify sSR as a predictor for survival and decreased incidence of thromboembolic events. This finding emphasizes the importance of identifying those patients in whom the restoration of sinus rhythm is an achievable goal and who should be given the opportunity to attain it. Our results suggest that appropriate patient selection should take into account left atrial dimensions, concomitant coronary heart disease, and the type of atrial fibrillation.

Concomitant myocardial revascularization was a negative predictor of maintenance in sinus rhythm. This association might reflect a more advanced stage of myocardial disease and difficulties in controlling arrhythmia in the presence of ischemia. These results are in contrast with the ones published by Prasad and colleagues. ${ }^{13}$

As previously reported, left atrial dimension was an independent predictor of sSR 1 year after surgical intervention. ${ }^{14}$ Our findings confirm that the likelihood of maintenance in sinus rhythm decreases as a continuum with increasing left atrial size, with a best cutoff point at $55 \mathrm{~mm}$. The presence of preoperative permanent atrial fibrillation was a negative independent predictor of sSR at 4 years but not at 1 year, where it was surpassed by the short-term predictors left atrial size and concomitant revascularization. According to our findings, patients undergoing multiple valve procedures should not be excluded as candidates for atrial fibrillation treatment because concomitant non-mitral valve surgery was not associated with a decrease in effectiveness. This might reflect selection bias but also the fact that patients requiring multiple valve procedures currently receive surgical treatment at much earlier stages than a few years ago.
Interestingly, a biatrial approach was an independent predictor for staying in SSR but was also associated with increased in-hospital mortality and the need for a permanent pacemaker. Further studies will be needed to identify the patients in whom the apparent benefit of this approach outweighs its potential risks. The greater effectiveness of biatrial approaches is not surprising. The highest reported rates of conversion to sinus rhythm belong to the maze procedure, ${ }^{9}$ a biatrial surgical technique. The importance of right atrial intervention is also underscored by the modest but instructive crude success rates $(8 \%-12 \%)$ of previous studies on catheter ablation with right atrial compartmentalization. ${ }^{10}$

The high prevalence of antiarrhythmic therapy postoperatively might also have contributed to the preservation of sinus rhythm during follow-up. Although the use of antiarrhythmic drugs might reflect an effort to keep these complex patients in sinus rhythm, it is probably magnified by the use of $\beta$-blockers and digoxin in the treatment of concomitant hypertension and chronic heart failure.

Several limitations of this study must be acknowledged. First, despite the large number of patients included in the registry, the sample size of this study is still suboptimal because an important proportion of patients were lost to follow-up during the first postoperative year and were excluded from further analysis. Because the data were collected retrospectively into a registry, patient selection and surgical technique were largely unstandardized. The use of 12-lead electrocardiography to establish rhythm during follow-up reflects the rhythm at a given moment and overestimates the success rate of these surgical procedures. Because treatment options were not randomized, differences in outcome between a biatrial approach versus a left atrial approach only and cut-andsew methods versus ablation methods might reflect selection bias rather than real differences in the safety and efficacy of surgical techniques. Before establishing a definite relationship between the achievement of SSR and improved outcomes, the effect of variables, such as cardiovascular risk factors that were not consistently recorded in this registry, should be taken into account.

In spite of the above limitations, these are encouraging results that require confirmation in large randomized controlled trials.

\section{References}

1. Geidel S, Ostermeyer J, Lass M, et al. Permanent atrial fibrillation ablation surgery in CABG and aortic valve patients is at least as effective as in mitral valve disease. Thorac Cardiovasc Surg. 2006;54:91-5.

2. Bando K, Kasegawa H, Okada Y, et al. Impact of preoperative and postoperative atrial fibrillation on outcome after mitral valvuloplasty for nonischemic mitral regurgitation. J Thorac Cardiovasc Surg. 2005;129: 1032-40.

3. Fuster V, Ryden LE, Asinger RW, et al. ACC/AHA/ESC guidelines for the management of patients with atrial fibrillation: executive summary. A Report of the American College of Cardiology/American Heart Association Task Force on Practice Guidelines and the European Society of 
Cardiology Committee for Practice Guidelines and Policy Conferences (Committee to Develop Guidelines for the Management of Patients With Atrial Fibrillation) Developed in Collaboration With the North American Society of Pacing and Electrophysiology. Circulation. 2001;104:2118-50.

4. Cox JL, Schuessler RB, D'Agostino HJ Jr, et al. The surgical treatment of atrial fibrillation: III. Development of a definitive surgical procedure. J Thorac Cardiovasc Surg. 1991;101:569-83.

5. Melo JQ, Adragão P, Neves J, et al. Surgery for atrial fibrillation using intra-operative radiofrequency ablation. Rev Port Cardiol. 1998;17: 377-9.

6. Knaut M, Spitzer SG, Karolyi L, et al. Intraoperative microwave ablation for curative treatment of atrial fibrillation in open heart surgery- the MICRO_STAF and MICRO_PASS pilot trial. Thorac Cardiovasc Surg. 1999;47:379-84.

7. Sie HT, Beukema WP, Ramdat Misier AR, Elvan A, Ennema JJ, Wellens HJJ. The radiofrequency modified maze procedure. A less invasive surgical approach to atrial fibrillation during open-heart surgery. Eur J Cardiothorac Surg. 2001;19:443-7.

8. Lee JW, Choo SJ, Kim KI, et al. Atrial fibrillation surgery simplified with cryoablation to improve left atrial function. Ann Thorac Surg. 2001;72:1479-83

9. Melo J, Adragao P, Neves J, et al. Endocardial and epicardial radiofrequency ablation in the treatment of atrial fibrillation with a new intraoperative device. Eur J Cardiothorac Surg. 2000;18:182-6.

10. Lima GG, Kalil RA, Leiria TL, et al. Randomized study of surgery for patients with permanent atrial fibrillation as a result of mitral valve disease. Ann Thorac Surg. 2004;77:2089-94.

11. Jessurun ER, van Hemel NM, Defauw JJ, et al. A randomized study of combining. The maze surgery for atrial fibrillation with mitral valve surgery. J Cardiovasc Surg. 2003;44:9-18.

12. Akpinar B, Guden M, Sagbas E, Sanisoglu I, Ozbek U, et al. Combined radiofrequency modified maze and mitral valve procedure through a port access approach: early and mid-term results. Eur J Cardiothorac Surg. 2003;24:223-30.

13. Prasad SM, Maniar HS, Camillo CJ, et al. The Cox maze III procedure for atrial fibrillation: long-term efficacy in patients undergoing lone versus concomitant procedures. J Thorac Cardiovasc Surg. 2003;126: $1822-8$.

14. Cappato R, Calkins H, Chen SA, et al. Worldwide survey on the methods, efficacy, and safety of catheter ablation for human atrial fibrillation. Circulation. 2005;111:1100-5.

\section{Discussion}

Dr Ralph J. Damiano (St Louis, Mo). I would like to congratulate Dr Melo for initiating this international registry and his coauthors for participating in this noble endeavor. As time progresses, I have no doubt it will yield very useful information and provide a broad look at current surgical practice for the treatment of atrial fibrillation. Already Dr Melo and his colleagues have assembled the largest reported series of concomitant mitral and atrial fibrillation surgery.

Although the registry does represent a major contribution to the field, it suffers from the shortcomings of all voluntary retrospective databases, and these shortcomings include selection and reporting bias and significant inhomogeneity of the patient populations, surgical skill, and technique and differing follow-up regimens between centers.

In my opinion the most significant finding of this study is that SSR was associated with better early and late survival and fewer thromboembolic events during the follow-up period. This has confirmed the work of Bando and others and adds further testimony to the crucial importance of performing atrial fibrillation surgery in patients who have chronic atrial fibrillation and are referred for the treatment of valvular heart disease.

Dr Melo, I have a number of questions for you. First, was there any attempt to standardize the follow-up between centers? For a patient to be considered to be either in SSR or SAF, were electrocardiographic or Holter monitoring data required to document the actual rhythm? If a patient had atrial flutter 6 months postoperatively and required an interventional procedure to terminate the flutter but then was in sinus rhythm at 1 year, would you consider that a failure or a success?

Dr Melo. Of course, this is a retrospective database, and therefore there was not the possibility of standardizing follow-up, but I should comment that if we have these activities to survive, we have to be more scientific. Therefore we need further time in putting together data prospectively instead of just to survive and to undoubtedly prove the things for which we are looking.

Regarding the information based on 12-lead electrocardiography or surface electrocardiography, which, of course, has the drawbacks of which we are all aware, at least there was an electrocardiogram to support that information.

Regarding patients who need to be cardioverted within the first semester and then remain in sinus rhythm, those patients would be classified as in sSR.

Dr Damiano. How about if they required an interventional catheterization to ablate atrial flutter, was that considered a success also if they went into sSR?

Dr Melo. That patient would be censored for this study because we have 2 or 3 patients. That patient represents a failure of surgical intervention, and therefore he needs to go to the catheterization laboratory for our goal of being very strict because, as you know, most reports, including Dr Bando's, are not talking about sinus rhythm. He is talking about regular rhythms, and most reports are talking about atrial fibrillation, which is a rather different issue.

Dr Damiano. My second question is that you had a wide range of ablation technologies in your study. Did you notice that ablation energy source had any effect on late results?

Dr Melo. That is the $\$ 1$ million question. We have to realize that unipolar radiofrequency, as you know, is less and less used, as opposed to actual analysis from bipolar and argon cryoablation, which are the energies that are currently being used. As you know, we had $4 \%$ and $5 \%$. Therefore we did not make comparisons between different forms of ablation energy, but compared instead cut-and-sew technique with ablation. We did not show any improved results using the cut-and-sew technique, even though we might have the shortcoming of having only 44 patients with the cut-and-sew technique, which is at this point maybe more critical to understand why most reports with the cut-and-sew techniques, which are maze techniques, apparently have slightly better results.

Dr Damiano. In a study that we published in 2005 on predictors of late recurrence after a full maze procedure, we found that the duration of preoperative atrial fibrillation was the strongest predictor of arrhythmia occurrence and operative failure. Did you find this to be a predictor of sSR in your database?

Dr Melo. No, and we looked thoroughly at that, and this is pretty much coincident with several articles published individually by several groups. It was not.

Dr Damiano. Finally, I was wondering whether you can explain the relatively poor late results of patients in your registry? The 1year freedom from atrial fibrillation was only $66 \%$, and if I have calculated from your manuscript correctly, the drug-free sSR rate at 1 year was only $44 \%$. This compares to certainly our own historical rate, where we have sSRs of more than $95 \%$ at 10 years, and $75 \%$ 
of those patients are drug free and in sinus rhythm, either a normal sinus rhythm or a paced rhythm. Even with ablation devices, we are presently getting more than $90 \%$ sSR at 1 year, and approximately $70 \%$ of patients are drug free. Do you believe that the worse results in this registry are due to the fact that $65 \%$ of patients only underwent left-sided procedures and you found that that was perhaps a risk factor for worse results? Also, of the left-sided procedures, three quarters of those patients only had pulmonary vein isolation, which many investigators, including myself, would believe would be an inadequate operation for patients with large left atria and organic heart disease?

Dr Melo. I think this is a very good question. I am not sure about the answer, even though from your question you are implying the answer. Number one, this is a pretty unselected patient population; left atrial size is, on average, $57 \mathrm{~mm}$. Therefore those are very sick patients, at least a large number of patients, which might have affected these results. On the other hand, I do concur with you and it is currently my practice that we should be performing biatrial approaches now on the basis of these data, and those are on the basis of our knowledge from the maze operation.

But more important than that, I think that you can dump, as you promised, your data in our database to compare apples with apples, which I am not sure we have been doing historically.

Dr Kevin D. Accola (Orlando, Fla). I congratulate the authors for putting together such a nice registry because it certainly helps clarify, with the array of energy sources, the results of this new technology. My question to you is about the cohort of patients who have had a pacemaker; you listed that you did have some, but you really did not elucidate that group of patients. Did this group of patients do better going from a chronic atrial fibrillation state to a paced atrial ventricular synchronous state? I would be interested in this because we are still experiencing, in some studies and in our practice, a $12 \%$ to $14 \%$ incidence of pacemaker insertion. Therefore I would be interested if you looked at this cohort individually and if these patients did better.

Dr Melo. We did look at that, and we have an incidence of pacemakers after left-sided approaches of $2 \%$, and we have an incidence of biatrial approaches, mazes plus whatever lines you do on the right side, at 1 year of $10 \%$. And those patients, if they had a sequential pacemaker, they were considered in sSR, but if they had only PVI, they would be atrial fibrillation patients. But I cannot give you in detail what has happened to that particular group of patients besides what I told you.
Dr Accola. As a follow-up, do you anticoagulate these patients for a particular duration, or what is your guidance to us on that?

Dr Melo. I think there is no evidence at the moment from whatever source that it is safe to interrupt anticoagulation on these patients, even if you close the left atrial appendage. Besides, you have very strong medical evidence that it is dangerous to stop anticoagulants because in patients undergoing mitral surgery, who are mostly rheumatic, there is very good evidence. The oldest one I am aware of is coming from the Mayo Clinic showing that of thrombi in lone atrial fibrillation, more than $90 \%$ start in the left atrial appendage, but in the context of rheumatic fever, half of thrombi are in the body of the left atrium because of the degenerative disease. Therefore I think that in most patients, especially if they are rheumatic, I do not believe it is safe to interrupt anticoagulants at this point.

Dr Guo-Wei He (Hong Kong, China). I congratulate you, Dr Melo, on your excellent presentation. My question is actually just to touch on your response to the previous discussant. My question is related to rheumatic heart disease. As you know, I am from China. We have a big problem with rheumatic heart disease. Most patients with mitral valve disease have a cause of rheumatic disease. Therefore I would like to know what is the incidence of rheumatic heart disease in this group? Furthermore, and more importantly, are there any differences among those patients with rheumatic heart disease after mitral surgery and with atrial fibrillation surgery who remain in sinus rhythm 1 year later compared with those who did not have atrial fibrillation surgery regarding the rate of sinus rhythm 1 or 2 years later in patients who had mitral surgery with rheumatic heart disease, the other group of that cause?

Dr Melo. As to the cause of disease of these patients, as I showed, around $60 \%$ had rheumatic fever. As far as it concerns these patients, if you are dealing with patients with very enlarged atria, very diseased atria, sometimes they do not even have myocytes, and I think it is not worthwhile to perform atrial fibrillation, especially because on those patients your success rate at 1 year, as we have reported 10 years ago, is in the range of $15 \%$. And now we know by this kind of analysis that it is eventually worse for a patient to be on an intermittent route than to be in atrial fibrillation. The worst scenario for those patients is the $4 \%$ or $5 \%$ of patients who, after these extensive procedures, instead of having a fibrillating atrium have a steady atrium, and I believe this is worse for thrombi. We still are treating patients with rheumatic fever. In those patients, if they have a large left atrium, we do not perform the operation. I think it is not worthwhile. 